Otfried Höffe

Philosophisches Seminar

Universität Tübingen

\title{
Minimalbürger, Staatsbürger, Weltbürger
}

Lange Zeit befaßte sich die Politische Philosophie vornehmlich mit sozialen Institutionen und Systemen. ${ }^{1}$ Die Politik erschien dabei als eine Auseinandersetzung mit Interessen und um Macht. Vernachlässigt wurden die Subjekte, von denen in Demokratien doch alle politische Gewalt ausgeht. Dieser Vernachlässigung steuert das Thema der Bürgeridentität entgegen. Das entscheidende Subjekt, den Bürger, darf man allerdings weder auf den Bürger im engeren Sinn, den Staatsbürger, verkürzen, noch bei diesem die Bürgertugenden vergessen. Selbst wer den Staatsbürger für den wichtigsten Aspekt hält, darf die anderen Hinsichten nicht unterschlagen, zumal sie das Staatsbürgersein beeinflussen. Die Bürgertugenden wiederum tragen als Rechtssinn, Gerechtigkeitssinn und Gemeinsinn zum Wohlergehen der Demokratie bei, gehören deshalb zum Kern eines aufgeklärten Liberalismus.

Die anglophone Diskussion gibt sich beim Bürgersein häufig mit zwei (Kymlicka/ Norman 1994), neuerdings drei Dimensionen zufrieden (Leydet 2011, Abschn. 1.1). Sie fängt beim Rechtsbürger an, dem bürgerliche, politische und soziale Rechte zukommen („legal status“). Sie setzt sich fort mit dem aktiven und wegen seiner Aktivität vorbildlichen Staatsbürger, der sich als "political agent" in den politischen Institutionen engagiert, worin Bürgertugenden anklingen. Und der neueren, dritten Dimension, geht es um jenes Mitglied einer politischen, identitätsstiftenden Gemeinschaft, den ich den kommunitären Bürger oder Gemeinschaftsbürger nenne.

Für die mittlere Dimension unterscheidet die anglophone Diskussion noch zwei Modelle, das die negativen Freiheitsrechte betonende, somit einen aufgeklärten Liberalismus verkürzende liberale Modell und das auf Aristoteles, Machiavellis Discorsi und Rousseau zurückgeführte republikanische Modell der staatsbürgerlichen Selbstregierung. (Dem Wortsinn nach steht freilich die Republik im Gegensatz zur Monarchie, weshalb die deutsche Rechtstradition dann vom Freistaat spricht.) Im republikanischen Modell gilt der Bürger vor allem als eine öffentliche

1 Der Artikel stellt einen Auszug aus dem kommenden Buch des Autors Kritik der Freiheit. Das Grundproblemm der Moderne dar. 
Person, als Citoyen, im (verkürzten) liberalen Modell dagegen als eine private, an Kommerz interessierte Person, als Bourgeois.

Für einen ersten Blick sind diese Begriffe zweifellos hilfreich, die nähere Betrachtung stößt auf deutliche Schwächen. Denn außer dem Staatsbürger, dem Citoyen, dem Wirtschaftsbürger, dem Bourgeois, und dem Gemeinschaftsbürger gibt es mindestens noch als vierte Person den Kulturund Bildungsbürger. Als Pfarrer, Lehrer, Hochschullehrer oder Journalist spielt er vermutlich nicht nur in Deutschland über viele Generationen eine tragende Rolle. Ferner greifen die drei Dimensionen von Bürgeridentität ineinander. Außerdem zeichnet sich die dritte, kommunitäre Dimension durch einen Facettenreichtum aus, so daß sie keine homogene Größe darstellt. Weiterhin halte ich für die ersten beiden Dimensionen eine andere Interpretation für sachgerechter. Nicht zuletzt lege ich auf eine fünfte Dimension, auf den Weltbürger, wert (s. Höffe 2004).

Wegen dieser Bedenken und Ergänzungen folge ich nicht der anglophonen Debatte, die zudem hier wie andernorts überwiegend nur mit sich beschäftigt ist. In (groß)provinzieller Selbstzufriedenheit nutzt sie ihre sprachliche Dominanz und nimmt, von übersetzten Klassikern abgesehen, kaum Debatten anderer Sprach- und Kulturräume zur Kenntnis.

Die politische Moderne beginnt historisch gesehen mit der Vision des aufgeklärten Bürgers (für einen differenzierteren Blick auf die Neuzeit s. Pinzani 2009; immer noch lesenswert Königsberg/Luckner 1988). Sie entwickelt sich fort oder präzisiert sich zur Vision des emanzipierten, später des mündigen Bürgers, der neuerdings im Gedanken des engagierten, namentlich partizipativen Bürgers kulminiert.

Die Identität des heutigen Bürgers an diesen Visionen auszurichten, also an der Aufklärung, einer facettenreichen Emanzipation und einer engagierten Partizipation, ist sinnvoll, da ein erheblicher Teil zeitgenössischer Identitätsfragen sich leicht darauf beziehen läßt. Diese Bezüge pflegt man allerdings auf aktuelle, häufig emotional aufgeladene Themen zu verkürzen. Dem gegenüber erlaubt sich diese Studie sowohl einen umfassenderen als auch hinsichtlich der aktuellen Debatten und genannten Visionen nüchterneren Blick. Aktuellen Schwierigkeiten verschließt sie sich allerdings nicht. Zusätzlich nimmt sie weitere, zum Teil grundlegendere Probleme wahr, auch wenn deren Wahrnehmung dem vorherrschenden Zeitgeist widerspricht. Ernsthafte Philosophie sucht zwar ihre Zeit auf den Begriff zu bringen, sie jedoch nicht auf einen modischen Zeitgeist zu verkürzen. 
Gemäß der methodischen Maxime sachgerechter Philosophie sôzein ta phainomena gliedere ich meine Überlegungen in sieben Schritte: Ich beginne mit einer Spannung im Leitbegriff (Abschn.). Gemäß einer weiteren methodischen Maxime, der Komplementarität von Abstieg und Aufstieg, führt danach ein methodischer Abstieg zu einer extremen Einschränkung der Identität, zum Minimalbürger (Abschn.). Seine Identität ist allerdings statisch und abstrakt, was im methodischen Aufstieg die Frage nach der konkret gelebten, in sich dynamischen Identität aufdrängt (Abschn.). Bei ihr kann die Religion zum Konfliktfall werden (Abschn.), woran ich Überlegungen zum interkulturellen Strafrecht (Abschn.) und drei Gestalten eines engagierten Staatsbürgers anschließe (Abschn.). Den letzten Schritt bildet die knappe, um eine Vermutung ergänzte Bilanz (Abschn.).

\section{Spannungen im Identitätsbegriff}

Auf den ersten Blick erscheint der Gehalt als klar und unstrittig. „Identität" bedeutet eine Gleichheit, sogar Selbigkeit. Allerdings tritt sie in zwei Stufen auf, zum einen als Artidentität oder, sozialtheoretisch gesehen, als Identität einer kleineren oder größeren Gruppe, als kollektive Identität, zum anderen als Identität der Zahl nach, als numerische Identität oder Identität einer natürlichen Person.

Darin liegt nun eine erste Komplikation. Die Frage nach der Identität eines Bürgers mag sich letztlich auf die zweite Stufe, auf die personale Identität eines Individuums, richten. Für sie sind aber kollektive Identitäten so wichtig, daß sich die personale Identität weitgehend als Bündel von kollektiven Identitäten bestimmen läßt. Dieses Bündel sieht freilich je nach Person unterschiedlich, darüber hinaus mehr oder weniger individuell aus. Mindestens in einen Teil der kollektiven Identität wird man hineingeboren, vor allem in ein Gemeinwesen oder, seltener in Staatenlosigkeit, ferner in einen gewissen Sprachraum und in eine Religionsgemeinschaft oder in die Nichtzugehörigkeit.

Derartige Identitätsfaktoren sind Vorgaben, mithin kein Gegenstand der persönlichen Freiheit und leisten paradoxerweise trotzdem einen konstruktiven Beitrag zur Freiheit. Als Bürger eines Staates genießt man dessen Schutz und erfreut sich zumindest in konstitutionellen Demokratien negativer und positiver Freiheitsrechte. Ohne eine Sprache oder eine wohlbestimmte Mehrsprachigkeit entwickelt man nicht die für ein reales Freiheitswesen notwendige Denk- und Kommunikationsfähigkeit. Und sofern eine Religionsgemeinschaft hilft, ein sinnvolles und rechtschaffenes 
Leben zu führen, stärkt sie die menschliche Freiheit, was allerdings Kritiker entweder einigen oder allen Religionsgemeinschaften abstreiten. Später, im Fortgang der Biographie, kann man sich mit den genannten Vorgaben auseinandersetzen und gegebenenfalls aus einem Gemeinwesen auswandern, einen Sprachraum wechseln oder in eine andere Religionsgemeinschaft eintreten oder auch aus jeder austreten. Ein Staat oder eine Religion, die dieses verbieten, schaffen erheblich Barrieren für die persönliche Freiheit und sind ihrerseits zutiefst unfrei.

In den modernen Gesellschaften des Westens dürfte das Maß an Individualität recht hoch, vermutlich höher als in vielen anderen Gesellschaften ausfallen. Bewahrheitet sich diese Hypothese, so hat sie die paradoxe Folge, daß kollektive Identitäten nicht nur die Art, sondern auch das Ausmaß an Individualität der Identität prägen. Aus diesem Grund empfiehlt sich eine weitere Distanz zur dominanten anglophonen Debatte: Die Bürgeridentität ist nicht global und allgemein, sondern in bezug auf den modernen westlichen Gesellschaftstyp zu erörtern. Hinzukommt, daß die zeitgenössische Globalisierung nicht bloß die Facetten der heutigen Bürgeridentität erweitert. Sie hat auch zu zwei diametral entgegengesetzten Entwicklungen geführt, die ein weiteres Problem schaffen: Vor allem in nichtwestlichen Gesellschaften tritt der einen Entwicklung, der globalen Annäherung der Gesellschaftsformen aneinander, eine Akzentuierung des Nichtglobalen, des Eigenen und Besonderen, entgegen. Mit dieser Gegenrichtung nimmt man sich ein Recht, ohne das die Globalisierung ein erhebliches Maß an Legitimität verlöre, man beansprucht ein Recht auf Differenz.

Dieser Umstand mahnt die im Westen dominanten Identitätsdebatten zu Bescheidenheit. Weil empirisch gesehen das Recht auf Differenz tatsächlich wahrgenommen wird, stellen sich in vielen nichtwestlichen Gesellschaften die Identitätsfragen auf andere Weise. Weil überdies, jetzt normativ betrachtet, die Wahrnehmung des Rechts unter wohlbestimmten Bedingungen berechtigt ist, sollten westliche Identitätsdebatten sich weder stillschweigend noch gar ausdrücklich als allgemein relevant gerieren. Sie sollten durch den Index „westlich“ ihre schon thematische Einschränkung anerkennen und sich für anders strukturierte, nichtwestliche Debatten offenhalten, mehr noch: Sie sollten interkulturelle Rechtsdiskurse führen.

Auch innerhalb des Westens ist das Recht auf Differenz zu respektieren. Bedenken erheben sich beispielsweise gegen die Neigung, die eigene kanadische Situation mit den für sie konstitutiven zwei Sprachräumen, der 
Anglophonie und der Frankophonie und zusätzlich den Ureinwohnern, den Eskimos, auf Gesellschaften zu übertragen, in denen grundlegende kulturelle Verschiedenheiten weitgehend nicht schon vor langer Zeit, sondern erst durch neuere Zuwanderung entstehen (gegen Tully 1995).

\section{Minimalbürger}

Selbst nur die westliche Bürgeridentität der Moderne ist ein verwickeltes und verzwicktes Phänomen. Rechtlich-bürokratisch gesehen basiert sie auf Elementen, die Rechtsdokumente wie der Personalausweis und der Paß enthalten und nicht für den Westen spezifisch sind. Von der dazu befugten Instanz ausgestellt, bescheinigen die Dokumente die Zugehörigkeit zum völkerrechtlich anerkannten Gemeinwesen, dem Staat. Diese rein rechtliche Zugehörigkeit macht den Bürger zum Staatsbürger. Dabei haben die weiteren Einträge - Paßbild, Geburtsdatum, Augenfarbe usw. - die Aufgabe, die betreffende Person als den angegebenen Staatsbürger zu identifizieren.

Nur in Klammern: Manche Bürger haben mehrere Pässe. Unter besonderen Umständen kann eine doppelte oder sogar dreifache Staatsbürgerschaft sinnvoll sein. Bei deren großzügiger Erlaubnis sollte man freilich vier Gesichtspunkte berücksichtigen. Erstens gibt es ein Gerechtigkeitsbedenken: Weil der Staatsbürger zu jener Instanz, dem Staatsvolk, gehört, von dem alle Staatsgewalt ausgeht, verdoppelt man bei doppelter Staatsbürgerschaft dieses basale Bürgerrecht, die Mitgliedschaft im politischen Souverän. Es ist ein Privileg, das wie alle Privilegien die Nichtprivilegierten diskriminiert. Nach einem zweiten Gerechtigkeitsargument kann sich eine Staatsbürgerschaft mit weiteren, nicht so basalen, gleichwohl erheblichen Vorrechten, etwa dem Erwerb von Immobilien oder Kunstgegenständen, verbinden, was den Inhaber mehrerer Staatsbürgerschaften zusätzlich privilegiert.

Nach dem dritten Gerechtigkeitsbedenken darf man sich die Schutzzusagen eines Landes aussuchen, sie gegebenenfalls sogar gegeneinander ausspielen. Und um Fall der Wehrpflicht kann man das Land auswählen, in dem man die Wehrpflicht ableistet, wobei einige sich die bequemere Art aussuchen, andere die Wehrpflicht in einem Land ableisten, dessen Militär sich auf eine vom anderen Land kritisierte Aktionen einläßt. Als viertes stellt sich die Identitätsfrage: Zuwanderer verdrängen die Aufgabe, mit dem neuen Land sich zu identifizieren. Nicht zuletzt droht die Gefahr, daß ausländische Politiker sich das Recht nehmen, in Reden und Aufrufen in die Innenpolitik des anderen Landes sich einzumischen. 
Und dieses muß man schon beschämend nennen: Ein so langfristig folgenreiches Thema wie die mehrfachen Staatsbürgerschaften wird nicht selten kurzfristigen parteipolitischen und koalitionstaktischen Vorteilen geopfert.

Zurück zur Paßidentität. Diese stellt eine Minimalidentität dar, die schon wegen ihrer Minimalität und zusätzlich, weil sie weit älter als die Globalisierung ist, die genannten gegenläufigen Entwicklungen der Globalisierung zu unterlaufen vermögen: Ohne das Recht auf Differenz zu beeinträchtigen, ist die Paßidentität eine global anerkannte Identität und sie gibt einen Grund mehr für das Recht ab, anerkanntes Mitglied eines Gemeinwesens zu sein und nicht als Staatenloser leben zu müssen.

Manche westliche Bürger mokieren sich zwar über ihre staatliche, gern als nationalstaatlich diskreditierte Identität. In unseren Breiten sind sie lieber nur Europäer, und im globalen Maßstab empfinden sie sich als bloße Weltbürger. Reisen sie aber in politisch gefährliche Gegenden, geraten dort in Gefangenschaft und werden nur gegen Lösegeld freigelassen, so hoffen sie nicht bloß, sondern erwarten staatliche Hilfe. Man nimmt also die genannte Schutzzusage in Anspruch. Selbstverständlich erfolgt die Schutzzusage von seiten des bislang diskreditierten Nationalstaates. Bei einer mehrfachen Staatsbürgerschaft wendet man sich am liebsten an den Staat, der in politischer, vielleicht zusätzlich in wirtschaftlicher Hinsicht mehr Erfolg verspricht. Jedenfalls neigt die Minimalidentität zu einer puren Forderungsidentität, nämlich zu einem Bürgersein, das sich auf Forderungen verkürzt.

Ebenfalls bloße Forderungen erhebt, wer zum Gemeinwesen außer erzwungenen Gegenleistungen wie Steuern nichts beiträgt. Der bloße Paßinhaber braucht jedenfalls keine emotionale Bindung an sein Land. Er hat keinerlei Pflicht, sich affirmativ oder kritisch mit der Geschichte, Tradition und Kultur, lange Zeit nicht einmal nachdrücklich mit der Amtssprache seines Landes verbunden zu fühlen.

Die Minimalisierung der Bürgeridentität kann noch weiter reichen. Der bloße Staatsbürger kann nach seinem Rechtsbewußtsein ein dem Staat fremdes Recht, beispielsweise die Scharia, für höherrangig halten. Bei schariainspirierten Rechtsverstößen muß er sich zwar vor den üblichen Kadi ziehen lassen, der seinerseits aber großzügig entscheiden kann. Der pure Staatsbürger braucht jedenfalls nicht einmal jenen Minimalpatriotismus zu pflegen, den Verfassungspatriotismus, den patriotismusskeptische Intellektuelle wie Jürgen Habermas 2009 im Anschluß an den 
Politikwissenschaftler Dolf Sternberger 1982 vertreten. (Neuerdings betont er aber das Eingebettetsein in eine historisch gewachsene Kultur.) Als Minimalbürger, selbst als bloßer Verfassungspatriot, ist jedenfalls der westliche Staatsbürger manchem positiven Pathos zum Trotz rechtlichpolitisch, noch mehr kulturell gesehen ein „Schrumpfbürger“.

Seine Identitätsschrumpfung kann noch weiterreichen. Die erwähnten zwangsbefugten Leistungen, die Steuern, ermöglichen dem Staat, die bekannten Gemeinwohlaufgaben zu finanzieren. Diese fangen beim Rechtsschutz an und reichen über die materielle Infrastruktur, also Kanalisation, Strom- und Gasleitungen, Straßen-, Eisenbahn- und Telekommunikationsnetzen, zum Gesundheitswesen, zum Bildungs- und Ausbildungswesen, nicht zuletzt zur Verteidigung und zu Beiträgen der Kultur. Bürger, die keine Steuern bezahlen, gelangen trotzdem in den Genuß all dieser Leistungen. Und in großzügigen Sozialstaaten können Bürger sogar, ohne jemals Steuern zu zahlen, ihr gesamtes Leben als Sozialhilfeempfänger führen. Die gelegentlichen Versuche, zumindest kommunale Dienstleistungen einzufordern, werden in der Regel rasch eingestellt.

\section{Dynamische Identität}

So weit fällt eine Skizze westlicher Bürgeridentität nicht bloß düster aus; sie ist auch offensichtlich unzureichend. Denn kaum ein Mensch kann mit einer derart verarmten und verkümmerten, ebenso statischen wie abstrakten Bürgeridentität leben. Der pure Rechtsbürger mag ein statischer Schrumpfbürger sein, auf den konkreten Menschen trifft die fulminante Attacke von Michel Foucault zu, die er in seiner Archäologie des Wissens $(1973,30)$ reitet und mit der er das weit größere Freiheitspotential der persönlichen Identität einfordert. „Man frage mich nicht, wer ich bin, und sage mir nicht, ich solle der gleiche bleiben: das ist eine Moral des Personenstands [d'état-civil]; sie beherrscht unsere Papiere. Sie soll uns freilassen, wenn es sich darum handelt, eine eigene Identität auszubilden.“

Die Hauptfigur in einem Roman von Fred Vargas, Das Orakel, nennt sich „Sohn des Rheins" und ist nicht auf eine Staatenlosigkeit, aber auf seine „unbestimmte Staatenlosigkeit“ stolz. Denn „der Vorteil, niemandem anzugehören, bestand darin, daß man jeder sein konnte“. Noch weiter geht, wer sich dagegen wehrt, in seinem souveränen Willen zur Ortsveränderung sich einem Papier, eben einem Paß, eventuell zusätzlich einem Visum, zu unterwerfen, um in andere Länder reisen zu können. Jedenfalls zeigt sich, daß selbst das Staatsbürgersein, aus dem elementare Rechte, 
mithin Freiheiten, folgen, nicht von Freiheitsgrenzen unbehelligt ist. Auch hier erweist sich die Freiheit als an Freiheitseinschränkungen gebunden.

Schon der Eintrag der Religions- oder Konfessionszugehörigkeit in der Lohnsteuerkarte mancher Länder zeigt die Gegenrichtung zum Schrumpfbürger an: Auf den Abstieg folgt der Aufstieg zum tatsächlich gelebten Bürgersein. Spätestens bei ihm erhält die Identität eine dynamische Komponente, die die Paßidentität nicht zu verändern braucht. Den Unterschieden, die bei den bald unmerklichen, gelegentlich eruptiven Prozessen konkreter Identitätsbildung virulent werden, liegt nun ein Merkmal westlicher Gesellschaften, der Pluralismus, zugrunde.

Bei Staaten wie Belgien, Indien, Kanada und der Schweiz, auch BosnienHerzegowina, die offiziell mehrsprachig sind, beginnen die Unterschiede bei der Amtssprache. Ein Schweizer ist franko-, germano- oder italophon oder spricht Rätoromanisch. Und Spanien hat eine einzige Staatssprache, Spanisch qua Kastilisch, in den jeweiligen autonomen Gemeinschaften läßt das Land aber Katalanisch, Galizisch und Baskisch als weitere Amtssprachen zu.

Während eine offizielle Mehrsprachigkeit nur in wenigen Ländern existiert, gibt es in vielen westlichen Staaten Zugehörigkeiten, die man dem Pluralismus zwar selten zuordnet, die aber häufig eine die Identität mitstiftende Bedeutung haben. Gemeint sind regionale Zugehörigkeiten teils politischer (Stichwort: Föderalismus), teils ethnischer Natur. Nehmen wir als Beispiel Deutschland: Das Land gliedert sich in Bundesländer wie Niedersachsen, Baden-Württemberg oder Bayern. Und in Baden-Württemberg, sagt schon der Name, leben zwei unterschiedliche Volksstämme, Badener und Schwaben. Letztere leben aber ebenso im Regierungsbezirk Bayrisch-Schwaben, womit sich auch Bayern als nach Volksstämmen verschieden erweist; außerdem gehören die Franken und die Oberpfälzer dazu.

Manche fühlen sich schon über die Sprachfärbung mit ihrer regionalen Herkunft verbunden, auch wenn sie längst anderswo, vielleicht sogar im Ausland wohnen. Andere wie der Autor haben eine kompliziertere Verbundenheit: In Oberschlesien geboren, in Westfalen aufgewachsen, habe ich vielerorts studiert, ein Jahrzehnt in München verbracht, zwischendurch ein Jahr in New York, später, von einem Berlin-Jahr unterbrochen, eineinhalb Jahrzehnte in der Schweiz verbracht und lebe nun schon mehr als zwei Jahrzehnte in Tübingen. Die Heimat für eine Biographie unter derartigen Bedingungen hat am ehesten vier Dimensionen, einmal die 
eigene Familie, zum weiteren den - bei mir: deutschen - Sprach- und Kulturraum, drittens dem Kern dessen, was Verfassungspatriotismus oder auch politischer Liberalismus meinen: den „Stolz“ auf Recht, Demokratie, Grundrechte und Toleranz, der mit dem eigenen Gemeinwesen beginnt und über seinen Großraum, hier: Europa, sich zu einem Kosmopolitismus öffnet, zu dem viertens und noch deutlicher staatenübergreifend der Beruf, die Philosophie, hinzukommt.

Zweifellos gehört nämlich in der heutigen Erwerbsgesellschaft der Beruf zu den identitätsstiftenden Zugehörigkeiten. Ferner fühlen viele Menschen sich mit anspruchsvollen Hobbys wie Sport, Schachspielen oder Musik verbunden, andere sind aktive Fans eines Fußballclubs. Wieder andere identifizieren sich mit nationalen oder internationalen Organisationen karitativer Natur wie Brot für die Welt, Misereor und SOS Kinderdörfer oder humanitärer Natur wie Ärzte ohne Grenzen, nicht zuletzt politischer Natur wie Amnesty International. Manche begnügen sich dabei mit Geldzuwendungen, andere engagieren sich aktiv, aber ohne Honorar, lassen sich also auf eine ehrenamtliche Tätigkeit ein.

Mit Sprache, Beruf und Hobbys hat begonnen, was die genannte dritte Dimension, den kommunitaristischen Identitätsbegriff, als entschieden zu eng zurückweist. Es gibt nämlich Staatengrenzen überschreitende Zugehörigkeiten und bei ihnen sowohl komplementäre als auch konkurrierende Identitäten. Eine Muttersprache hat man in der Regel nur eine; allerdings wachsen manche Menschen so gut wie perfekt zweisprachig, einige wenige sogar dreisprachig oder noch mehrsprachiger auf. Vor allem kann man seine Leitsprache wechseln bis hin zu so spektakulären Fällen wie dem Polen Korzeniowski, den wir unter seinem Vornamen Josef Conrad als englischen Schriftsteller kennen. Sein anglophoner Kollege William Somerset Maugham wächst zunächst frankophon auf. Vladimir Nabokov schreibt anfangs auf Russisch, später gelegentlich auf Französisch, mehrheitlich aber auf Englisch. Und der Mongole Galsan Tschinag hat das Deutsche um seine sprach- und gefühlssensiblen Erzählungen und Romane wie Dojnana und Die neun Träume des Dschingis Khan bereichert.

Die sprachliche Konkurrenz aufzuheben, scheint weder möglich noch sinnvoll zu sein, denn sie kann Kreativitätskräfte freisetzen. Man sollte ihr jedoch den Stachel der Gewaltbereitschaft ziehen. Zusätzlich darf man in Europa von jedermann, weltweit zumindest von allen Intellektuellen, auch Politikern und anderen führenden Personen erwarten, daß sie eine Fremdsprache zu sprechen, eine zweite Fremdsprache verstehen. Denn mitlaufend lernt man, was in van Parijs 2013 Plädoyer für das Englische 
als Weltsprache unterbewertet, für Zeiten der Globalisierung aber mehr als nur willkommen zu heißen ist: Man lebt sich in eine andere Kultur ein, die man in der Regel auch zu schätzen und in seine Argumente einzubauen lernt.

Sprachen sind nicht nur ein Mittel der Kommunikation. Weit mehr als bloß ein Instrument der Zirkulation von Gütern, Dienstleistungen und Kapitel, auch weit mehr als nur ein Mittel, um Informationen, Ansichten und Überzeugungen zu artikulieren, haben sie in einem emphatischen Sinn des Wortes einen Bildungswert. Sie bieten den Einstieg in neue Denkwelten; sie lassen Andersartigkeit erfahren und helfen, den geistigen Reichtum Europas mit seiner Fülle von hochentwickelten Kultursprachen wahrzunehmen (s. dazu Trabant 2014).

Im übrigen trifft zu, was ein britischer Bibliothekswissenschaftler einmal in Paris bemerkte: Trotz des enormen Konformitätsdrucks seitens des Englischen erscheinen in Europa immer noch die Mehrheit wissenschaftlicher Publikationen auf Deutsch.

\section{Konfliktfall Religion}

In einem relevanten Faktor des Pluralismus, der Vielfalt von Religionen und Konfessionen oder aber Religions- bzw. Konfessionslosigkeit, sind die westlichen Gesellschaften noch pluralistischer geworden. In vielen muslimisch dominierten Ländern dagegen, in der lange Zeit eine Multireligiösität herrschte, sind die religiösen Minderheiten empfindlich bedroht. Ein eindrucksvolles Gegenbeispiel, die in Bosnien-Herzegowina vorher gelebte, geradezu vorbildliche religiöse Toleranz zwischen Muslimen, Katholiken, orthodoxen Christen und Juden, wurde durch den Krieg von 1992-1995/96 empfindlich gestört, ging aber glücklicherweise nicht verloren.

Im Westen gab es lange Zeit außer der Vielfalt der christlichen Konfessionen eine jüdische Minderheit, während die wenigen Muslime eine exotische Ausnahme bildeten. Die später wachsende Zahl von Buddhisten blieb unauffällig; Bhagwan- Anhänger und Scientology erfuhren nur gelegentliche Aufmerksamkeit. Die heute auffallendere religiöse Pluralisierung geht allein auf die Zuwanderung von Muslimen zurück.

Erst in dieser Zuwanderung findet sich ein Bündel von Andersartigkeiten, die beispielsweise über die der jüdischen Gemeinden weit hinausreichen. Das Andere kann mit der Kleidung, dem Kopftuch oder sogar der Burka, beginnen, obwohl auch orthodoxe Juden, sowohl Männer als auch Frauen, 
besondere Kleidungsvorschriften beachten. Es kann sich im Schächten, also einem von den Tierschutzbestimmungen abweichenden Schlachten, zeigen, und muß noch nicht bei Gebeten außerhalb der Gebetsräume, in der Öffentlichkeit, enden. Manche Muslime haben nämlich Schwierigkeiten damit, daß die Mädchen beim üblichen Sport-, besonders Schwimmunterricht, teilnehmen, vielleicht sogar damit, daß sie eine Freundschaft mit Nichtmuslimen eingehen. Hinzukommt die Neigung zu einer Paralleljustiz durch muslimische Friedensrichter, und besonders kritikwürdig sind die sogenannten Ehrenmorde.

Derartige Andersartigkeiten bilden nicht notwendig den Bestandteil einer muslimischen Bürgeridentität. Denn einerseits gibt es wie im Judentum und Christentum so auch im Islam viele Richtungen, überdies sowohl strenggläubige als auch liberale, nicht zuletzt ihrer Religion weithin entfremdete Muslime. Überdies sind die auffallenden Andersartigkeiten nur zum Teil genuin religiöser Natur, so daß man bei ihnen nicht von einer muslimischen Bürgeridentität, sondern nur von einer Identität muslimischer Bürger sprechen kann. Und diese fällt wegen der angedeuteten innermuslimischen Unterschiede nicht homogen aus.

Sofern die angedeuteten Unterschiede nicht genuin religiöser Natur sind, handelt es sich um Gewohnheiten und Mentalitätsdifferenzen, die vom wirtschaftlichen und sozialen Umfeld der Herkunftsregion abhängen. Nicht erst in Berlin, Basel, Madrid oder Rom werden Zuwanderer aus ostanatolischen Dörfern als fremd wahrgenommen, sondern schon in vielen Vierteln türkischer Großstädte wie Istanbul. Und in Sarajevo, wo mittlerweile etwa $80 \%$ der Bevölkerung Muslime sind, sieht man auf den Straßen nur wenige Frauen mit Schleier und werden die in den letzten Jahren neuen, von außen finanzierten Moscheen mit ihren auffallenden Minaretten als architektonische Fremdkörper empfunden.

Notwendigerweise konkurrieren Religionszugehörigkeiten übrigens nicht. Ein Japaner kann Shintoist und Buddhist, zusätzlich sogar Christ sein so wie Christen den Zen-Buddhismus praktizieren können. Judentum, Christentum, von dessen erster, oft jüdischen Generation abgesehen, und Islam dagegen, auch Islam und Hinduismus schließen sich genauso gegenseitig aus wie Katholizismus und Protestantismus oder Sunniten- und Shiiten-Zugehörigkeit.

Beide, die religiöse und die konfessionelle Konkurrenz, haben sich oft in blutigen Kämpfen ausgetobt; selbst heute hat der religiöse Faktor der Identität mancherorts noch einen polemogenen Einschlag. Dem gegenüber ist 
Toleranz zu verlangen, womit in die Identitätsfrage ein normatives Moment hereinkommt: Bürger haben sich gegenseitig als freie und ebenbürtige Personen anzuerkennen, die das Recht auf eigene Überzeugungen haben und nach ihnen sollen leben dürfen. Dazu gehört das Recht, als Staatsbürger seine Identität von der Religionszugehörigkeit her mitzubestimmen, aber auch die Pflicht, anderen das Recht auf eine davon unabhängige, eventuell sogar religionsfeindliche Identität einzuräumen.

\section{Interkulturelles Strafrecht}

Daß die Toleranz dort endet, wo die Gegenseite Rechte und Freiheit anderer mißachtet, dabei sogar gewalttätig wird, versteht sich. Namentlich bei Rechtsfragen stößt die Toleranz berechtigterweise auf Grenzen. Der Hinweis auf die Sharia zeigt allerdings exemplarisch, daß nicht alle

216 Einwohner, seien sie eingebürgert oder nicht, die Grenzen anerkennen. Der Grund kann in Identitätsproblemen liegen, die beim Recht, namentlich dessen einschneidenster Form, dem Strafrecht, besonders gravierend ausfallen. Die Probleme sind zwar nicht an die Globalisierung gebunden, erhalten in Zeiten der Globalisierung aber eine neue Aktualität.

Die einschlägige Frage, ob eine Rechtsordnung das (subjektive) Recht hat, ihr (objektives) Strafrecht kulturübergreifend anzuwenden, habe ich andernorts untersucht (s. Höffe 1999). Im Blick auf die Bürgeridentität sei lediglich an zwei Gesichtspunkte erinnert. Zum einen ist bei Elementen kollektiver Identität, weit mehr als nur das Wohlbefinden, nämlich der Freiheitskern der personalen Identität, das Recht auf die eigene Religion, die eigene Sprache und die eigene Kultur, betroffen. Vor allem wird, wer nicht schon in jungen Jahren auswandert, zu einer identitätsgeschädigten Person, die weit eher zur Kriminalität neigt als eine identitätsgefestigte. Zum anderen hat die Frage nach einem interkulturellen Strafrecht mit dem Feindbild des „gefährlichen Fremden“ nichts zu tun.

Ein interkulturelles Recht, nicht nur Strafrecht, läßt sich nach fünf Antwortmustern rechtfertigen: (1) nach dem Prinzip der Gleichbehandlung, (2) mit einem interkulturellen Recht wie dem römischen ius gentium, (3) mit einem kulturindifferenten Strafrecht, das den Menschen schlicht als Rechtsperson behandelt, (4) mit einem (straf-)rechtlichen Föderalismus und (5) der Behandlung nach dem Herkunftsrecht (Höffe 1999, Kap. 2).

Am stärksten überzeugt eine Rechtfertigung aus der universalistischen Rechtsmoral der Menschenrechte. Hier versteht sich das Strafrecht als Schutzschild der möglichen Opfer und beansprucht genau deshalb eine 
interkulturelle Gültigkeit. Die Freiheitseinschränkung, die es den möglichen Tätern aufzwingt, sichert nämlich den potentiellen Opfern Vertrauen und die dadurch gespeiste Freiheit.

Ein menschenrechtsgestütztes Strafrecht schützt beispielsweise Delinquenten vor Leibesstrafen; es toleriert keine sogenannten Ehrenmorde. Auch bewahrt es (junge) Frauen vor Zwangsheiraten, ferner vor Bestrafung von Ehebruch und vor dem - angeblichen - Recht, sie zu Opfern sexueller Übergriffe zu machen. Identitätsschwierigkeiten sollte das Einhalten der entsprechenden Verbote keinem Zuwanderer („Migranten“) bereiten. Ist es trotzdem der Fall, so ist deren Bewältigung sowohl von den Wortführern der zuwandernden Gruppen als auch von jedem einzelnen Individuum einzufordern. Nicht das geringste Argument dafür liegt in dem Hinweis, daß schon im Herkunftsland der Zuwanderer keine Menschenrechtsverletzungen erlaubt sein dürften. Und im Gastland rechtfertigen Identitätsprobleme Menschenrechtsverletzungen gewiß nicht.

Die angedeuteten Probleme sind in der Regel primär nicht individueller, sondern kollektiver Natur, daher nicht leicht zu bewältigen; unlösbar sind sie aber auch nicht. Sollte das mit den Menschenrechten konfligierende Recht einen religiösen Hintergrund haben, was aber wenig wahrscheinlich ist, so tragen die im Gastland aktiven religiösen Gruppen und Vereine ein hohes Maß an Mitverantwortung. Das Gastland-Gemeinwesen hat hier nicht bloß das Recht, sondern sogar die Pflicht, die Mitverantwortung einzufordern: im gesellschaftlichen Umgang, in den Medien, als ultima ratio auch über das zwangsbefugte Recht. Nach meiner persönlichen Erfahrung haben die westlichen Gesellschaften diese Aufgabe mindestens zwei, drei Jahrzehnte verschlafen. Dadurch haben sich Mentalitäten verfestigen können, die jetzt schwieriger zu ändern sind. Diese Erschwernis erlaubt aber nicht, die Aufgabe weiterhin zu verdrängen; sie zu bewältigen erfordert jedoch größere Anstrengungen.

\section{Wutbürger, Gemeinwohlbürger, Weltbürger}

Sprache und Religion reichern die Bürgeridentität an, die sich, wie angedeutet, in einer regionalen Zugehörigkeit und der Verbundenheit mit Berufsgruppen und Hobbys verdichten kann. Andere Faktoren leisten mehr als eine Anreicherung und Verdichtung, denn sie steigern ein bescheidenes, schlichtes Bürgersein zu einem anspruchsvollen Staatsbürgersein. Dessen Minimum besteht in der Anerkennung von Recht, Demokratie, Grundrechten und Toleranz, ein mehr als Minimum in der Teilnahme an Wahlen und dem vorangehenden Sich-Vertraut-Machen mit politischen 
Entwicklungen und Programmen. Und die veritable Steigerung manifestiert sich in einem aktiven „Stolz“ auf eine konstitutionelle Demokratie und freiheitliche Gesellschaft, nämlich im diesbezüglichen Engagement.

Sichtbar wird es in der Übernahme von Ehrenämtern oder in öffentlichen Wortmeldungen oder auch der Mitwirkung in Bürgerinitiativen, worin manch aktiver Staatsbürger sogar seine wichtigste Identität sieht. Bürgerinitiativen bewegen sich im Zwischenbereich zwischen privaten und öffentlichen Interessen. Ihr bürgerschaftliches Engagement, ein wichtiger Faktor im republikanischen Bürgerverständnis, wendet sich gegen einen etatistisch verkürzten Politikbegriff. Allerdings darf man sie nicht mit dem gleichsetzen, was die größere Aufmerksamkeit der Medien auf sich zieht, mit den „Wutbürgern“. Zweifellos stiftet deren Tätigkeit Identität, und für manche mag die wöchentliche Demonstration ein Gottesdiens-

218 tersatz sein, ein Aspekt, mit dem eine pluralistische Gesellschaft keine Schwierigkeit hat. Bedenken tauchen erst dann auf, wenn „Wutbürger“ ihrem Interesse an Medienpräsenz ein so kostbares politisches Gut wie die Rechtsstaatlichkeit opfern.

Schon aus diesem Grund darf man Bürgerinitiativen nicht grundsätzlich positiv einschätzen. Hinzukommt, daß sie sich nicht notwendig für das Gemeinwohl einsetzen, denn auch der Protest gegen Infrastrukturmaßnahmen wie Stromtrassen, Eisenbahnlinien, auch Müllversorgungsanlagen kann von einem zwar verständlichen, aber doch partikularen Interesse getragen sein. Selbst dort, wo Gemeinwohl-Interessen verfolgt werden, besteht die Gefahr, einen kleinen Teil des Gemeinwohls zu privilegieren, beispielsweise in der Rentendebatte die Gerechtigkeit gegen künftige Generationen herunterzuspielen.

Der unvoreingenommene Blick nimmt jedenfalls das Bürgerengagement nicht nur in Bürgerinitiativen, sondern ebenso im reichen Strauß von Freiwilligenarbeit und Ehrenämtern wahr oder darin, daß sich jemand öffentlich zu Wort meldet. Zugleich widerspricht er einem liberalismuskritischen Kulturpessimismus, demzufolge die Bürger wohlhabender Demokratien egoistische Monaden seien, die nichts als ihr Selbstinteresse verfolgen. In Wahrheit gehört für viele stark ausgeprägte Individuen die Mitwirkung in gemeinnützigen Vereinen, Verbänden und Selbsthilfegruppen, die ehrenamtliche Betreuung von Randgruppen und Benachteiligten oder die Arbeit in Bürgerinitiativen, kurz: ein soziales und politisches Engagement zu ihrer Bürgeridentität hinzu.

Das Gemeinwesen, auf das sich das aktive Bürgerengagement richtet, muß nicht im Staat, es kann auch in kleineren Einheiten, in der Kommune, 
im Bundesland, Kanton oder der Provinz, nicht zuletzt im globalen Gemeinwesen einer Weltgesellschaft bestehen. Der entsprechend engagierte Bürger, der Gemeinwohlbürger, zeichnet sich, worauf ein aufgeklärter Liberalismus großen Wert legt, durch Bürgertugenden aus, die mit dem Rechtssinn beginnen und über den Gerechtigkeitssinn samt Zivilcourage schließlich im Gemeinsinn ihr relatives Maximum finden.

Die bescheidenste Bürgertugend, der Rechtssinn, gibt sich mit einer Rechtstreue zufrieden, die entweder aus Angst vor Strafen oder aus freien Stücken die geltenden Gesetze befolgt. Die mittlere Stufe, ein Gerechtigkeitssinn, votiert legislatorisch für den Abbau von Privilegien und Diskriminierungen und applikativ für eine unparteiische Anwendung von Gesetzen, auch gegen das hemmungslose Ausnützen von Schlupflöchern. Sieht der Gerechtigkeitssinn massives Unrecht gegeben, so behält er sich, sofern er couragiert genug ist, einen (staats-)bürgerlichen Ungehorsam vor. Freilich droht die Gefahr, daß partikulare Interessen hereinspielen oder daß man die im Rechtsstaat üblichen Oppositionsmittel scheut. Um dieser Gefahr einer Selbstprivilegierung zu entgehen, unterwirft sich ein ernsthafter bürgerlicher Ungehorsam einem Aufrichtigkeitstest, nach dem man Nachteile in Kauf zu nehmen bereit ist.

Eine normativ erneute Steigerung von Bürgeridentität erlangt, wer einen Gemeinsinn praktiziert. Dessen erste, in republikanischen Theorien meist vernachlässigte Gestalt, der kulturelle Gemeinsinn, sucht das eigene kulturelle Kapital, einschließlich der eigenen Sprache, den künftigen Generationen mindestens ebenso reich zu hinterlassen, wie man es ererbt hat. In Zeiten der Ökonomisierung sprechen für diese Leistung sogar ökonomische Gründe. Wohin ein Wissenschaftler eine Berufung annimmt oder wohin ein Konzern seine Europazentrale stellt, hängt nämlich auch vom „Handelswert“ der Landessprache und von der kulturellen Infrastruktur des zuständigen Ortes ab. Letztlich kommt es dem kulturellen Gemeinsinn aber nicht darauf, sondern auf den Eigenwert von Sprache und Kultur an.

Bei der Praxis der zweiten Gestalt, bei dem gemeinnützigen Aufgaben verpflichteten sozialen Gemeinsinn, ist der Wert so offensichtlich, daß er keiner näheren Darstellung bedarf. Nur der genannte Aspekt sei wiederholt: Die kulturpessimistische Ansicht, liberale Bürger seien atomisierte Egoisten, wird durch die Wirklichkeit kräftig widerlegt.

Der politische Gemeinsinn schließlich, der Gemeinsinn als Staatsbürgersinn, hat im Unterschied zu den meisten republikanischen Theorien ein doppeltes, nicht bloß innen-, sondern auch außenpolitisches Gesicht. 
Im Inneren bemüht er sich um ein hohes Maß an Partizipation in seinem Gemeinwesen, das er wegen dessen politischem Liberalismus hochschätzt und nach außen um einen umsichtigen Ausgleich von Selbstwertgefühl und Fremdschätzung. Als ein aufgeklärter Patriotismus vermeidet er zwei Fehlformen, sowohl ein nationalistisches Selbstlob als auch eine Idiophobie, die aus Angst vor dem Xenophobievorwurf das Eigene nur zu mißachten oder zu kritisieren versteht und sich nicht einmal gegen unqualifizierte Angriffe von Ausländern aus Selbstachtung wehrt.

Eine unverkürzte Bürgeridentität kennt zwei weitere Dimensionen: Viele sich selbst kritisch nennende Intellektuelle pflegen die Sphäre der Wirtschaft zu verachten, allerdings häufig mit dem pragmatischen Widerspruch, daß sie alimentiert sein, darüber hinaus finanziell gut dastehen wollen. Die Wirtschaft ist aber nicht nur persönlich, sondern auch für das Gemeinwohl unerläßlich. Ihre Unverzichtbarkeit beginnt mit den Arbeitsplätzen, die in der heutigen Arbeits- und Berufsgesellschaft von existentieller Bedeutung sind und sich dabei keineswegs auf den Aspekt des Lebensunterhalts beschränken, sondern auch die fachliche Ausbildung, ferner Sozialkompetenzen, außerdem eine interkulturelle Kompetenz, ohnehin die Chance zu Anerkennung und zur Selbstachtung einschließt. Dabei kommt der sozialen Achtung eine besondere Bedeutung zu, denn sie und nicht etwa das Einkommen unterscheidet die Schichten (Sennett/Cobbe 1972). Die soziale Achtung darf allerdings nicht auf die öffentliche Reputation verkürzt werden. Nicht minder wichtig ist die Achtung in der Familie, durch Nachbarn, Freunde und Berufskollegen. Und beide, die große, öffentliche und die kleinere, mehr private Achtung können einander widerstreiten.

Eine florierende Wirtschaft ist nicht zuletzt deshalb gemeinwohlverpflichtet, weil sie jene Steuereinkünfte erhöht, die zur Finanzierung der Parlamente und der Justiz, des Gesundheits- und des Bildungswesens, des Sozialstaates, kultureller Einrichtungen und der materiellen Infrastruktur nötig sind. Wer als Unternehmer oder als Arbeitnehmer zum wirtschaftlichen Wohlergehen beiträgt, braucht sich dieses Aspekts seiner Bürgeridentität, seines Erfolges als Wirtschaftsbürger, nicht zu schämen.

Die zweite noch fehlende Dimension ist eher gut beleumdet; die Ausdrücke "Weltbürger" und Kosmopolit" haben einen guten Klang. Für eine kluge Bürgeridentität sind sie allerdings nur dann sinnvoll, wenn man sie nicht exklusiv, sondern komplementär versteht, also nicht als Ablehnung des Staatsbürgerseins, sondern als dessen Ergänzung. 
Schon wegen staatenübergreifender Gemeinsamkeiten wie Sprache, Religion und Berufstätigkeit, ferner wegen des vielfältigen wirtschaftlichen, wissenschaftlichen, politischen und kulturellen Austausches, weiterhin wegen des globalen Handlungsbedarfs, etwa hinsichtlich Verbrechensbekämpfung und Klimaschutz, nicht zuletzt wegen der universalistischen Rechts- und Staatsprinzipien begnüge man sich nicht mit der traditionellen Rolle des zwar partizipatorisch engagierten, aber mit dem eigenen Gemeinwesen zufriedenen Bürgers. Vielmehr erweitere man dort, wo erforderlich, den Tätigkeitsbereich aller drei Stufen, des Rechtssinns, des Gerechtigkeitssinns und des Gemeinsinns, auf überregionale, schließlich auf globale Aufgaben.

Diese Erweiterung obliegt nicht bloß den Jetset-Eliten. Ob als Tourist, Konsument oder Arbeitnehmer, ob als Zeitungsleser, an Kultur Interessierter oder als Student und Dozent, nicht zuletzt über eine multiethnische Schüler- und Arbeiterschaft sowie das elektronische Weltnetz („Internet") und das großstädtische Erscheinungsbild - so gut wie jeder westliche Bürger erlebt fast täglich globalisierte Lebensverhältnisse.

\section{Bilanz und eine Vermutung}

Mit diesen Hinweisen kann die Skizze zur westlichen Bürgeridentität schließen und trotz ihrer Vorläufigkeit eines deutlich machen: Die Bürgeridentität ist ein komplexes Phänomen, dessen abstrakter und statischer Minimalkern, der Schrumpfbürger, sich im konkret gelebten Bürgersein um ein Bündel zahlreicher Faktoren anreichert. Die genaue Zusammensetzung ändert sich zwar im Laufe des Lebens; gelebte Identität ist ein dynamisches Phänomen, das im Zuge des Erwachsenwerdens und eines erfahrenen Lebens in der Regel reicher wird. Dabei pflegen für Zuwanderer, aber auch für Heimische interne Konflikte aufzutreten, bei deren Lösungsversuchen die Identität sich zu verändern pflegt. Bei Brüchen in der Biographie kann die Identität bis zu einem fast vollständigen Verlust erodieren. Der „Verfassungspatriotismus“ oder „patriotische Liberalismus" zu nennende Kern sollte aber davon untangiert bleiben.

Der angedeutete dynamische Prozeß drängt eine Frage auf, die für einen aufgeklärten Liberalismus wichtig ist und mit deren tentativer Antwort die Skizze schließt: Ist der Bürger bei der Suche nach seiner Identität frei? Meine tentative Antwort: Zweifellos hängt man von kollektiven Identitäten seiner Herkunft und des Umfeldes ab, in dem er lebt. Zur Bürgeridentität, insbesondere zur Identität des Staatsbürgers einer konstitutionellen Demokratie, gehört aber zweierlei, einmal daß man diese 
Demokratie mehr als nur verbal schätzt, zum anderen daß er im Rahmen dieser Wertschätzung sich selber und seine Mitbürger - Kinder und Unmündige ausgenommen - als für ihr Tun und Lassen verantwortliche Personen betrachtet. Aus diesem doppelten Grund, daß wir die konstitutionelle Demokratie für unseren unveräußerlichen Lebensrahmen halten und daß wir innerhalb dieses Rahmens dem Menschen eine Verantwortung für sein Leben zusprechen, halten wir die Bürger für an ihrer Identitätsbildung mitverantwortlich. Dabei können die genannten Bürgertugenden, der Rechtssinn, der Gerechtigkeitssinn und der Gemeinsinn, als Lebenseinstellungen mit Vorbildcharakter dienen.

Primljeno: 25. avgusta 2014.

Prihvaćeno: 27. avgusta 2014.

Literatur

222 Foucault, M. (1973), Archäologie des Wissens, Frankfurt a.M.: Suhrkamp.

Höffe, O. (1999), Demokratie im Zeitalter der Globalisierung, München: Beck.

Höffe, O. (2004), Wirtschaftsbürger, Staatsbürger, Weltbürger: Politische Ethik im Zeitalter der Globalisierung, Münich: Beck.

Kymlicka, W., \& Norman, W. (1994), „Return of the citizen: A survey of recent work on citizenship theory", Ethics 104: 352-381.

Leydet, D. (2011), „Citizenship“, The Stanford Encyclopedia of Philosophy (Fall 2011 Edition), Edward N. Zalta (ed.).

van Parijs, P. (2013), Sprachengerechtigkeit, Frankfurt/M.

Pinzani, A. (2009), An den Wurzeln moderner Demokratie: Bürger und Staat in der Neuzeit (Vol. 18), Berlin: Akademie Verlag.

Sennett, R., \& Cobb, J. (1972), The Hidden Injuries of Class, New York: VintageBooks.

Trabant, J. (2014), Globalesisch, oder was?: ein Plädoyer für Europas Sprachen, Münich: Beck.

Tully, J. (1995), Strange Multiplicity. Constitutionalism in an Age of Diversity, Cambridge, MA. 